\title{
Aspectos prácticos para la clasificación, diagnóstico y manejo de hiponatremia en el paciente hospitalizado
}

\author{
Harvey Julián Mejía-Sandoval* \\ Rafael Castellanos-Bueno** \\ Diego Alejandro Rangel-Rivera*** \\ Karen Lorena Rangel-Rivera****
}

\begin{abstract}
*Médico internista. Departamento de Medicina Interna. Universidad Industrial de Santander. Bucaramanga, Colombia.
**Médico internista. Especialista en Endocrinología y Nutrición. Grupo GERMINA. Departamento de Medicina Interna. Profesor Asistente. Universidad Industrial de Santander. Bucaramanga, Colombia.

****Residente de Medicina Interna III año. Grupo GERMINA. Departamento de Medicina Interna. Universidad Industrial de Santander. Bucaramanga, Colombia.

****Estudiante de medicina V año. Universidad Industrial de Santander. Bucaramanga, Colombia.

Correspondencia: Dr. Diego Alejandro Rangel Rivera. Dirección: Calle 32 \# 32-70, apartamento 901B. Bucaramanga, Santander, Colombia. Correo electrónico: diegorangelrivera@gmail.com
\end{abstract}

Resumen

La hiponatremia es el trastorno hidroelectrolítico más frecuente observado en pacientes hospitalizados y es importante resaltar que se ha asociado a morbilidad y mortalidad de estos. Esta entidad representa un proceso fisiopatológico relacionado con una alteración en la homeostasis del agua, en la cual los pacientes presentan síntomas en su mayoría neurológicos, que se correlacionan con el nivel de sodio y el tiempo de aparición del trastorno. Se realizó una búsqueda en las bases de datos PubMed y Lilacs de monografías, artículos de revisión y artículos originales con el objetivo de revisar aspectos sobre la clínica, diagnóstico y manejo de la entidad. Para el diagnóstico, la batería de estudios se solicita en función del contexto clínico, actuando temprano y permitiendo la corrección del trastorno de acuerdo al escenario respectivo. La hiponatremia es un problema médico frecuente que bajo un abordaje práctico y sencillo permite tomar decisiones clínicas de forma oportuna. MÉD.UIS. 2020;33(2):85-93.

Palabras clave: Hiponatremia. Electrólitos. Sodio. Solución salina. Agua corporal.

\section{Practical aspects for the classification and management of hyponatremia in the hospitalized patient}

Abstract

Hyponatremia is the most frequent hydroelectrolytic disorder observed in hospitalized patients and it is important to note that it has been associated with morbidity and mortality in this patients. This entity represents a pathophysiological process related to an alteration in water homeostasis, in which patients present symptoms mostly neurological, that correlate with the sodium level and the time of onset of the disorder. A search of the PubMed and Lilacs databases of monographies, review articles and originals articles was performed with the objective to review clinical, diagnostic and management aspects of this entity. For diagnosis, the clinical laboratory studies are requested depending on the clinical context, acting early and allowing correction of the disorder according to the respective scenario. Hyponatremia is a frequent medical problem that requires a practical and simple approach favoring clinical decisions in a timely manner. MÉD.UIS. 2020;33(2):85-93.

Keywords: Hyponatremia. Electrolytes. Sodium. Saline solution. Body Water.

¿Cómo citar este artículo?: Mejía-Sandoval HJ, Castellanos-Bueno R, Rangel-Rivera DA, RangelRivera KL. Aspectos prácticos para la clasificación, diagnóstico y manejo de hiponatremia en el paciente hospitalizado. MÉD.UIS. 2020;33(2):85-93. doi: 10.18273/revmed.v33n2-2020009

Artículo recibido el 21 de junio de 2020 y aceptado para publicación el 27 de agosto de 2020 


\section{Introducción}

La hiponatremia se define como una concentración sérica de sodio menor a $135 \mathrm{mEq} / \mathrm{L}$, siendo este el trastorno hidroelectrolítico más frecuente observado en pacientes hospitalizados'. La frecuencia de esta condición varía de acuerdo al escenario clínico donde se evalúe ${ }^{2}$. Un análisis con 5179 pacientes mayores de 55 años de la cohorte del estudio Rotterdam, encontró que en consulta externa la prevalencia de hiponatremia puede llegar hasta un $7 \%$ mientras en los servicios de urgencias puede alcanzar valores cercanos al $42 \% 3$. Su prevalencia en salas generales es del $6 \%$, mayor en pacientes críticos $(17,2 \%)$ y en unidades de geriatría $(22,2 \%)^{4}$. En cuanto a distinción por sexo, la hiponatremia se presenta más en mujeres, en parte por factores hormonales ${ }^{5}$. Cabe resaltar que los datos de epidemiología en este contexto son escasos y, por lo tanto, no se dispone de cifras nacionales adecuadas. En estudios observacionales se ha descrito que condiciones como diabetes mellitus, enfermedad renal crónica, fragilidad, falla cardíaca y el uso de diurético se comportan como factores de riesgo para el desarrollo de hiponatremia ${ }^{4,6}$.

Este trastorno no representa una enfermedad en sí misma, sino un proceso fisiopatológico relacionado con una alteración en la homeostasis del agua. Por ende el "paciente con hiponatremia" no existe y siempre que el clínico se enfrente a un paciente con niveles bajos de sodio, su labor será encontrar la condición subyacente que está originando la hiponatremia?. En ocasiones no se cuenta con una guía de manejo para estos pacientes, por lo que es pertinente una revisión con aspectos clave de su fisiopatología, diagnóstico y, por supuesto, un manejo apropiado.

En cuanto a la fisiopatología hay que tener en cuenta que la concentración de sodio sérico refleja el contenido de agua y sodio en la porción acuosa del plasma y el volumen circulante total7. Por lo tanto, existe una regulación a través de sistemas integrados que involucran osmorreceptores, barorreceptores y sistemas de respuesta a través de mecanismos neurohumorales?.

Se deben revisar algunos conceptos que, si bien están correlacionados, se deben diferenciar. En primer lugar, la osmolalidad se refiere a la cantidad de osmoles por kilogramo (valor normal: 275 a 295 $\mathrm{mOsm} / \mathrm{kg}$ ); la osmolaridad se refiere a la cantidad de osmoles por litro de agua plasmática (valor muy similar a osmolalidad) (valor normal: 275-290 mOsmol/L); y la tonicidad que se refiere a la concentración de osmoles efectivos, es decir aquellos que no pueden atravesar de forma libre las membranas ${ }^{7}$ (Ver Tabla 1). Por esta razón, los principales determinantes de la tonicidad plasmática van a ser la glucosa y el sodio'.

Tabla 1. Fórmulas relevantes para cálculos en hiponatremia

\begin{tabular}{|c|c|}
\hline Parámetro & Ecuación \\
\hline Osmolalidad (mOsmol/kg) & 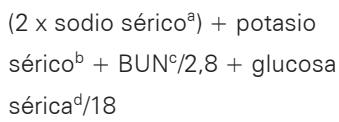 \\
\hline Tonicidad (mOsmol/L) & $\begin{array}{l}\left(2 \times \text { sodio sérico }^{a}\right)+\text { potasio } \\
\text { sérico }^{b}+\text { glucosa séricad }^{d} / 18\end{array}$ \\
\hline Osmolaridad urinaria ${ }^{8}(\mathrm{mOsm} / \mathrm{kg})$ & $\begin{array}{l}\text { (Sodio urinario }^{\mathrm{a}}+\text { potasio } \\
\left.\text { urinario }^{\mathrm{b}}\right) \times 2+\text { (Úrea en } \\
\left.\text { orina }^{\mathrm{e}} / 5,6\right)\end{array}$ \\
\hline
\end{tabular}

a Sodio en $\mathrm{mEq} / \mathrm{L}$; ${ }^{b}$ Potasio en $\mathrm{mEq} / \mathrm{L}$; ${ }^{\mathrm{c}} B U N$ en $\mathrm{mg} / \mathrm{dL}$; ${ }^{\mathrm{d}} \mathrm{Glucosa}$ en $\mathrm{mg} / \mathrm{dL}$; eÚrea en $\mathrm{mg} / \mathrm{dL}$

Fuente: autores

En la regulación del sodio se ven involucrados componentes como renina, angiotensina, aldosterona, péptidos natriuréticos y el sistema simpático ${ }^{7}$. Estos sistemas se activan principalmente frente a cambios en la presión arterial, perfusión renal y concentración de sodio sérico, modificando la actividad en los canales sensibles a amilorida localizados en la nefrona distal y la reabsorción de sodio'.

La principal hormona involucrada en la regulación de la tonicidad es la vasopresina (u hormona antidiurética) ${ }^{9}$, ésta se sintetiza a nivel de los núcleos supraóptico y paraventricular del hipotálamo, pero se acumula en la porción posterior de la hipófisis. Los principales estímulos para su liberación incluyen una reducción de la presión arterial, aumento de la osmolalidad y el aumento de la concentración de sodio. No obstante, algunas situaciones como el dolor, el estrés y los procedimientos quirúrgicos también aumentan su liberación ${ }^{9,10}$. Esta hormona actúa en los receptores V2 a nivel de la membrana basolateral de las células principales del túbulo colector, favoreciendo la expresión a nivel de la membrana apical de las acuaporinas 2, lo que permite el ingreso de agua a las células mencionadas. Luego, por efecto de la médula hipertónica (obtenido por el sistema de contracorriente) se genera un flujo de agua por medio de acuaporinas constitutivas en la membrana basal hacia el intersticio y finalmente al espacio intravascular?. 
Con lo anterior, se puede afirmar que el aumento en la liberación de vasopresina produce una elevación de la osmolalidad de la orina, reducción de la tonicidad sérica y de la concentración de sodio. De hecho, en algunas situaciones patológicas donde la perfusión efectiva es baja (tales como falla cardíaca y cirrosis), se genera liberación de vasopresina independiente del nivel de sodio lo que conduce a hemodilución e hiponatremia7. Es importante resaltar que la medición de vasopresina no se realiza de rutina, por lo que la osmolalidad urinaria puede ser usada como subrogado de este valor dado su correlación directa (un aumento de vasopresina conduce a elevación de la osmolalidad urinaria y viceversa $)^{1,11}$.

La importancia de la hiponatremia radica en su papel como factor pronóstico relacionado con morbilidad y mortalidad en una cantidad importante de entidades clínicas. La reducción de sodio sérico se ha asociado desenlaces deficientes en cuanto a sobrevida en falla cardíaca, cirrosis hepática, enfermedad renal crónica y cáncer ${ }^{12,13}$. Sin embargo, existen controversias en cuanto a esta asociación dado que si bien hay una relación entre la hiponatremia y mortalidad, no hay una asociación demostrada con el grado de severidad de la primera ${ }^{14}$. Asimismo, existen autores que dudan si realmente la hiponatremia es un factor causal del mal pronóstico o realmente corresponde a un marcador de enfermedad avanzada, sumado al hecho de la poca evidencia de mejoría en los parámetros funcionales tras la corrección del trastorno en las enfermedades crónicas'.

Es primordial un actuar oportuno y certero de acuerdo al contexto clínico. Por ejemplo, en los casos crónicos y asintomáticos habrá más tiempo para intervenciones y seguimiento, mientras que un caso agudo y sintomático demandará reposición urgente de sodio, vía endovenosa ${ }^{15}$. Por lo anterior, conocer los aspectos fundamentales de esta entidad, desde fisiopatología hasta manejo, es un gran apoyo para elegir el camino a seguir más adecuado, minimizando los errores. Este trabajo se ha realizado con el objetivo de hacer mención de los aspectos claves en la práctica clínica, con una propuesta de los aspectos más relevantes acerca del abordaje diagnóstico y terapéutico de la hiponatremia en el paciente hospitalizado.

\section{Metodología de búsqueda}

La estrategia de búsqueda de información se realizó en las bases de datos Pubmed y Lilacs durante los meses comprendidos entre septiembre de 2019 y marzo de 2020. Se utilizó la siguiente combinación de los términos: ((hyponatremia[MeSH Terms]) AND (physiopathology)); ((hyponatremia[MeSH Terms]) AND (classification)); ((hyponatremia[MeSH Terms]) AND (therapy)).

Se incluyeron monografías, artículos de revisión y artículos originales, en idiomas inglés y español, con fecha de publicación de los últimos cinco años a la búsqueda. Los artículos debían tener posibilidad de lectura de resumen, para poder incluir aquellos que trataran aspectos fisiopatológicos y clínicos de hiponatremia, excluyendo los que no hablaran de conductas diagnósticas o terapéuticas para los pacientes en el ámbito hospitalario. Con esta búsqueda se obtuvieron un total de 30 artículos que aportaban la información que se busca plantear en esta revisión de tema, de los cuales fueron excluidos 6 por ser duplicados o por idioma diferente a los mencionados. Finalmente, fueron revisados 24 artículos para la inclusión, extracción y redacción del manuscrito final.

\section{Desarrollo del tema}

\section{Manifestaciones clínicas}

Los síntomas de hiponatremia usualmente se correlacionan con el nivel de sodio y el tiempo de aparición del trastorno. La mayoría de los síntomas inician con niveles de sodio menores a $130 \mathrm{mEq} / \mathrm{L}$, pero algunos síntomas neurocognitivos han sido descritos con valores mayores ${ }^{16}$. Esto principalmente en población susceptible como los adultos mayores frágiles y pacientes con enfermedad renal crónica en hemodiálisis, en los cuales se ha relacionado la hiponatremia con un mayor riesgo de deterioro cognitivo, demencia y caídas ${ }^{17,18,19}$. Debe destacarse que muchos pacientes pueden ser asintomáticos o tener manifestaciones leves como cefalea y alteraciones del estado de ánimo ${ }^{20}$.

A medida que disminuyen los niveles de sodio, se acentúan las manifestaciones neurológicas, llevando a alteraciones del estado mental, pasando inicialmente por desorientación, confusión y finalmente síntomas más severos como convulsiones, obnubilación y coma ${ }^{21}$.

El tiempo de instauración de los síntomas también determina la presentación clínica. Los cuadros más 
severos, que amenazan la vida ocurren usualmente en las primeras 48 horas, como consecuencia de un edema cerebral de instauración rápida, que no permite que se den los mecanismos de adaptación para aminorar este proceso. Como consecuencia fatal puede haber herniación transtentorial y muerte por compresión del tallo cerebral, con afección de los centros cardiorespiratorios ${ }^{22}$.

Se presenta en la tabla 2, la relación entre los síntomas de hiponatremia y algunos aspectos relevantes de su clasificación clínica ${ }^{16}$ (Ver Tabla 2).

Tabla 2. Relación entre los síntomas y la clasificación de la hiponatremia

\begin{tabular}{|c|c|c|c|}
\hline Severidad & $\begin{array}{l}\text { Niveles } \\
\text { séricos de } \\
\text { sodio }\end{array}$ & Síntomas & $\begin{array}{l}\text { Duración } \\
\text { típica }\end{array}$ \\
\hline Leve & $\begin{array}{l}\text { Generalmente } \\
\text { menor a } 135 \\
\mathrm{mEq} / \mathrm{L}\end{array}$ & $\begin{array}{l}\text { Cefalea, irritabilidad, } \\
\text { problemas de } \\
\text { concentración, } \\
\text { alteración del estado } \\
\text { de ánimo, depresión. }\end{array}$ & Crónica \\
\hline Moderada & $\begin{array}{l}\text { Generalmente } \\
\text { menor a } 130 \\
\mathrm{mEq} / \mathrm{L}\end{array}$ & $\begin{array}{l}\text { Náuseas, vómito, } \\
\text { calambres, } \\
\text { hiporreflexia, } \\
\text { confusión, } \\
\text { desorientación, } \\
\text { alteración del estado } \\
\text { mental, inestabilidad } \\
\text { para la marcha. }\end{array}$ & $\begin{array}{l}\text { Aguda o } \\
\text { crónica }\end{array}$ \\
\hline Severa & $\begin{array}{l}\text { Generalmente } \\
\text { menor a } 125 \\
\mathrm{mEq} / \mathrm{L}\end{array}$ & $\begin{array}{l}\text { Convulsiones, } \\
\text { obnubilación } \\
\text { depresión respiratoria, } \\
\text { coma. }\end{array}$ & Aguda \\
\hline
\end{tabular}

Fuente: autores.

\section{Clasificación}

Al ser una condición heterogénea, existen múltiples formas de clasificar la hiponatremia de acuerdo a diferentes criterios incluyendo la severidad, la duración, la ocurrencia de síntomas, la osmolaridad y la volemia del paciente. La importancia de una correcta clasificación radica en el establecimiento de un tratamiento oportuno y adecuado, según cada caso particular. En la figura 1 se describen las diferentes clasificaciones de la hiponatremia que pueden ser usadas en el abordaje inicial7 (Ver Figura 1).

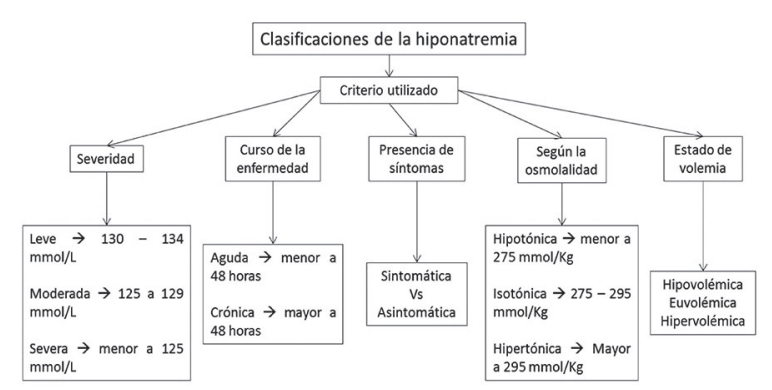

Figura 1. Clasificaciones de la hiponatremia

Fuente: autores.

Aproximación según la osmolaridad

\section{a: Pseudohiponatremia o hiponatremia isoosmolar}

La pseudohiponatremia o hiponatremia isoosmolar se presenta como una hiponatremia con osmolaridad normal habitualmente en condiciones como hipertrigliceridemia, dislipidemia o paraproteinemia?. El origen de este valor incorrecto de sodio se debe a que la mayoría de equipos asume una distribución normal del componente acuoso y no acuoso del plasma (93\% como agua). En las situaciones antes mencionadas, hay reducción de la fracción del volumen acuoso del plasma por lo que la cantidad de sodio es menor ${ }^{1}$. La utilización de potenciómetros directos (como los equipos de gases arteriales) no tienen este problema y permiten verificar la verdadera concentración de sodio.

\section{b: Hiponatremia hiperosmolar}

La hiponatremia junto con tonicidad plasmática mayor a $295 \mathrm{mOsm} / \mathrm{kg}$ sugiere el diagnóstico de hiponatremia hiperosmolar, asociado a hiperglucemia, uso de manitol o medios de contraste yodados 7 . En estos casos, la presencia de hipertonicidad favorece el movimiento de agua hacia el medio extracelular por lo que la concentración de sodio se reduce. Se describe que un aumento de 100 $\mathrm{mg} / \mathrm{dL}$ de glucosa sérica se traduce en la reducción de 1,6 mEq/L de sodio sérico ${ }^{23}$.

\section{c. Hiponatremia hipoosmolar}

Existen varias recomendaciones del enfoque de la hiponatremia hipoosmolar. Un enfoque sencillo involucra la utilización de la osmolalidad urinaria para determinar el correcto aclaramiento renal de agua 
(osmolalidad urinaria menor a $100 \mathrm{mOsm} / \mathrm{kg}$ ) junto con la medición del sodio sérico (Ver Figura 2). De esta manera, permite clasificar en algunos subgrupos para mejor entendimiento':

- Exceso de ingesta de fluido hipotónico con aclaramiento normal de agua a nivel renal: se presenta en los pacientes con ingesta de una cantidad elevada de líquidos hipotónicos en un volumen mayor a la capacidad de eliminación de agua libre a nivel renal, dando como resultado una hiponatremia dilucional. Se presenta en pacientes con polidipsia primaria, uso excesivo de líquidos hipotónicos parenterales o irrigación de líquidos hipotónicos en procedimientos como colonoscopia o procedimientos de urología. En este caso, la presencia de una historia clínica con orina diluida (osmolalidad menor a $100 \mathrm{mOsm} /$ kg) sugieren el diagnóstico'.

- Alteración del aclaramiento del agua sin cambio en la ingesta de líquidos: este escenario corresponde a pacientes en los cuales no hay una reducción de la liberación de la vasopresina frente a un plasma hipoosmolar (por lo tanto, la osmolalidad urinaria es mayor $100 \mathrm{mOsm} / \mathrm{kg})^{1}$. En este caso, se presenta un fenómeno de hiponatremia y hemodilución. El caso típico corresponde al síndrome de secreción inadecuada de hormona antidiurética, secundario a una larga lista de medicamentos (por ejemplo, amiodarona, carbamazepina, algunos antipsicóticos, diuréticos y antidepresivos) y como consecuencia de algunos tipos de cáncer ${ }^{24}$.

- Alteración del aclaramiento renal de agua con ingesta inapropiada de fluidos hipotónicos: en este caso, sucede una combinación de situaciones donde hay un aumento de liberación de vasopresina no mediada por factores relacionados a la osmolalidad plasmática. Este fenómeno sucede posterior a procedimientos quirúrgicos o en atletas con recorridos de largos trayectos, donde una reposición inadecuada de líquidos junto la elevación propia de la hormona antidiurética conduce a hiponatremia. Es también el caso para pacientes con falla cardíaca, cirrosis o diuréticos'1.

\section{Evaluación diagnóstica}

Realizar una evaluación ordenada, por pasos, permite tener un diagnóstico certero y evitar errores en el manejo. A continuación, se presenta un algoritmo de estudio desarrollado a partir de los hallazgos de esta revisión (Ver Figura 2).

Paso 1. Excluir hiponatremia no hipotónica y pseudohiponatremia. Debe excluirse la hiperglucemia como una de las causas principales de hiponatremia no hipotónica, así como el uso de manitol o radiocontraste hipertónico. Para confirmar que se trata de una hiponatremia no hipotónica, puede medirse la osmolalidad sérica. Si se sospecha pseudohiponatremia, en condiciones como hipertrigliceridemia o hiperproteinemia, se sugiere la medición del sodio a través de un potenciómetro directo o en gases arteriales, para evitar el artefacto de laboratorio que ocurre en estos $\operatorname{casos}^{1,25}$.

Paso 2. Evaluar la temporalidad y la presencia de síntomas severos. Si la hiponatremia se ha desarrollado en menos de 48 horas o si hay presencia de síntomas severos como convulsiones, alteración del estado de consciencia (obnubilación, coma) o dificultad respiratoria, debe iniciarse tratamiento inmediato con solución salina hipertónica (ver sección de tratamiento)'.

Paso 3. Evaluar la presencia de un defecto de dilución de la orina. Para esto es necesario medir o calcular la osmolalidad urinaria (Ver Tabla 1). Esto permite diferenciar causas mediadas por la vasopresina $(\mathrm{ADH})$ de las independientes de este mecanismo. Si la osmolalidad urinaria es menor a $100 \mathrm{mOsm} /$ $\mathrm{Kg}$, se descarta un defecto en la dilución de la orina (mecanismo independiente de ADH) y debe pensarse en causas como polidipsia primaria, baja ingesta de solutos o potomanía de la cerveza. Si por el contrario la osmolalidad urinaria es mayor a $100 \mathrm{mOsm} / \mathrm{Kg}$, el mecanismo es mediado por ADH y debe continuarse en el algoritmo diagnóstico ${ }^{1,25}$. La obtención de la osmolaridad urinaria, debe realizarse idealmente a través de medición directa con un osmómetro, siendo más precisa, o calculada a través de diferentes fórmulas, siendo menos exacta e inválida bajo ciertas circunstancias ${ }^{8}$.

Fórmula 1: se tiene en cuenta la densidad urinaria (DU) y la del agua (=1). Se obtiene multiplicando los 2 últimos valores de la densidad urinaria por $35^{8}$.

$$
\operatorname{Osm}(\mathrm{mOsm} / \mathrm{Kg})=(\mathrm{DU}-1000) \times 35
$$

Se deben hacer correcciones en la DU si hay glucosuria y/o proteinuria. Por cada $\mathrm{g} / \mathrm{dl}$ de 
glucosuria, disminuir la DU en 0,004 y por cada g/dl de proteinuria, descender la DU en 0,003. La fórmula se invalida cuando se ha usado previamente manitol, piperacilina, carbenicilina o carbapenémicos ${ }^{8}$.

Fórmula 2: se obtiene determinando los electrolitos y la urea en orina. Debe descartarse la presencia de glucosuria para que tenga valide $z^{8}$.

Osm $(\mathrm{mOsm} / \mathrm{Kg})=($ sodio urinario + potasio urinario $)$ $\times 2+$ (urea en orina / 5,6)

Paso 4. Evaluación del volumen arterial efectivo. Para esto se recomienda la medición del sodio en orina, dado que la determinación por evaluación clínica ha demostrado tener una baja sensibilidad $(50-80 \%)$ y especificidad $(30-50 \%)^{26,27}$. Si este es menor a $30 \mathrm{mmol} / \mathrm{L}$, indica una respuesta renal a un estado de hipovolemia o un bajo volumen arterial efectivo.

Deben tenerse en cuenta las siguientes consideraciones acerca de la medición del sodio en orina: puede estar bajo en personas con dietas hiposódicas, el uso previo de diuréticos puede aumentar sus niveles y los pacientes con enfermedad renal crónica puedentener concentraciones mayores al tener una menor capacidad de reabsorción de sodio. Se resalta que el bajo volumen arterial efectivo puede verse tanto en condiciones con aumento de fluido extracelular como falla cardíaca, cirrosis o síndrome nefrótico y en condiciones con disminución de fluido extracelular como pérdidas por tracto gastrointestinal, tercer espacio, o uso de diuréticos ${ }^{1,25}$.

Paso 5. Determinar uso previo de diuréticos. Si el sodio en orina es mayor a $30 \mathrm{mmol} / \mathrm{L}$ debe evaluarse el uso previo de diuréticos, siendo esta la principal causa si el antecedente es positivo. De lo contrario deben tenerse en cuenta otras causas basados en la evaluación del nivel de fluido extracelular ${ }^{1,25}$.

Paso 6. Evaluación clínica del líquido extracelular. Si este se encuentra disminuido deben considerarse como causas la insuficiencia adrenal primaria, el cerebro perdedor de sal, pérdidas renales de sodio y de origen gastrointestinal. Si el líquido extracelular esta normal, la causa más frecuente es el síndrome de secreción inadecuada de hormona antidiurética, encontrándose también la insuficiencia adrenal secundaria y el hipotiroidismo $0^{1,25}$.
Paso 7. Considerar uso oculto de diuréticos. Finalmente, si la evaluación de las causas anteriormente mencionadas es negativa, debe tenerse en cuenta el uso inadvertido de diuréticos como etiología de la hiponatremia ${ }^{1,25}$.

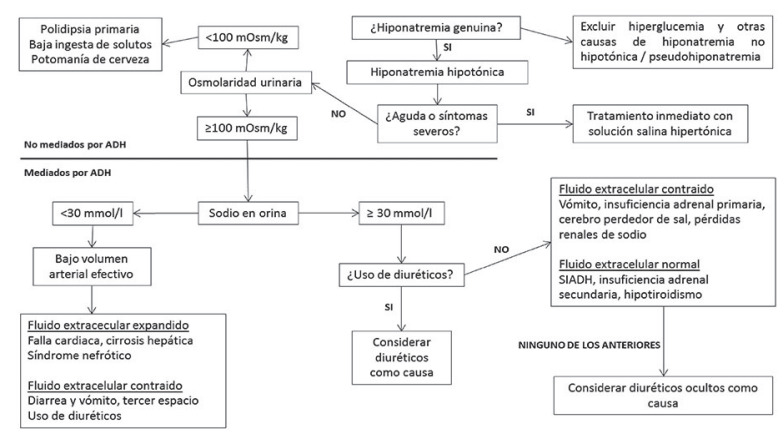

Figura 2. Evaluación diagnóstica de la hiponatremia

Fuente: autores.

\section{Tratamiento}

El tratamiento del paciente con hiponatremia depende de la velocidad de instauración ${ }^{28}$. En especial, los pacientes con casos leves crónicos que cursan con una corrección rápida tienen riesgo elevado de Síndrome de Desmielinización Osmótica (SDO) ${ }^{7,9}$. En general, se debe asumir que el paciente cursa con hiponatremia crónica (excepto para los pacientes hospitalizados, donde se puede verificar el tiempo real de evolución) ${ }^{29}$. También, es importante tomar en cuenta la clasificación y etiología para elegir el mejor tratamiento.

En los casos de hiponatremia hipertónica secundario a hiperglucemia, se deben utilizar medidas para el descenso de la glucosa junto con una reposición de líquidos endovenosos para corregir la depleción de volumen?. En casos de hiponatremia isotónica se debe descartar paraproteinemia y dislipidemia para definir el manejo específico de dichas condiciones. En los pacientes con hiponatremia hipotónica, se debe tener en cuenta el grado de volemia del paciente y el mecanismo que la produjo para definir la conducta terapéutica ${ }^{23}$.

Cuando se está corrigiendo el sodio sérico, la tonicidad alrededor del tejido del sistema nervioso central aumenta también, por lo cual existe riesgo de deshidratación y muerte celular secundaria. Este fenómeno se conoce como SDO, suele generar síntomas 3-4 días después del evento y causando un compromiso neurológico variable con secuelas irreversibles ${ }^{30}$. También, se han identificado algunos 
factores de riesgo como: concentración de sodio menor a $105 \mathrm{mEq} / \mathrm{L}$, consumo crónico de alcohol, desnutrición y enfermedad hepática avanzada ${ }^{31}$. Por lo anterior, es importante establecer metas seguras de corrección del sodio con el objetivo de mantener al paciente en un rango terapéutico, pero sin aumentar el riesgo de SDO.

El uso de solución hipertónica al 3\% (SS3\%) sólo se indica en presencia de niveles muy bajos de sodio o frente manifestaciones neurológicas graves como convulsiones o edema cerebral'. En este escenario, se plantea el uso de bolos de SS3\% de 100-150 cc cada 10-20 minutos (máximo 3) en búsqueda de un ascenso de sodio en la primera hora de $4-6 \mathrm{mEq} /$ $\mathrm{L}^{32}$. Posteriormente, se debe indicar una infusión intravenosa para garantizar un ascenso continuo. Hay diversas fórmulas descritas para la preparación de solución hipertónica, pero algunas de ellas no aportan un grado real cercano al 3\%. En la tabla 3 se resumen algunas de ellas para su consideración, resaltando especialmente el uso de las dos primeras (Ver Tabla 3).

Si bien existen diferentes fórmulas para el cálculo de la infusión, todas son ecuaciones estáticas frente a un proceso complejo y dinámico como lo son éstos pacientes. Por lo anterior y teniendo en cuenta la experiencia propia, se considera pertinente el uso de una infusión de 0,5-1 mL/kg/hora de SS3\% (que en promedio tiene una elevación de sodio de 0,5-1 mEq/L/ hora) con control estricto de sodio sérico cada 4-6 horas. Las metas de ascenso estándar corresponden a no más de $12 \mathrm{mEq} / \mathrm{L}$ en las primeras 24 horas, para luego continuar con un ascenso diario entre 6-8 $\mathrm{mEq} / \mathrm{L}^{7}$. Unos cortes más bajos, especialmente en las primeras 24 horas se pueden plantear en los pacientes con mayor riesgo de SDO.

\section{Escenarios especiales}

Algunas medidas y consideraciones adicionales basadas en hallazgos de la literatura que se deben tomar en cuenta para el paciente con hiponatremia hipotónica, según la volemia del paciente, se presentan a continuación:

\section{a. Hiponatremia hipervolémica}

Se debe evaluar primero la causa bien sea falla cardíaca o cirrosis hepática, en cuyo caso el tratamiento se basa en las restricción de agua libre y de sal ${ }^{28,32}$. Asimismo, el uso de diuréticos de asa suele hacer parte del tratamiento. Se ha planteado el uso de vaptanes pero se debe tomar en cuenta los efectos de algunos de ellos como el conivaptan (riesgo de vasodilatación esplácnica) o el tolvaptán (riesgo de hepatotoxicidad)1. Por lo tanto, sólo se recomienda para una minoría de pacientes que son refractarios al tratamiento de primera línea.

Tabla 3. Fórmulas descritas en la literatura para solución hipertónica, concentración, gramos y porcentaje aproximado resultante.

\begin{tabular}{|c|c|c|c|}
\hline $\begin{array}{l}\text { Forma de } \\
\text { preparación (mEq } \\
\text { de sodio) }\end{array}$ & $\begin{array}{l}\text { Concentración } \\
\text { de sodio por } \\
\text { litro de solución }\end{array}$ & $\begin{array}{l}\text { Gramos } \\
\text { de soluto } \\
\text { (sodio + } \\
\text { cloro) por } \\
\text { litro }\end{array}$ & $\begin{array}{l}\text { Porcentaje } \\
\text { de } \\
\text { solución } \\
(\%)\end{array}$ \\
\hline $\begin{array}{l}\text { Agua destilada } \\
370 \mathrm{cc}+130 \mathrm{cc} \\
\text { ( } 260 \mathrm{mEq} \text { ) Cloruro } \\
\text { de sodio al } 10 \%\end{array}$ & $520 \mathrm{mEq} / \mathrm{L}$ & 30,4 & 3 \\
\hline $\begin{array}{l}\text { SS 0.9\% } 405 \text { cc } \\
(62 \mathrm{mEq})+95 \text { cc } \\
(190 \mathrm{mEq}) \text { Cloruro } \\
\text { de sodio al 10\% }\end{array}$ & $504 \mathrm{mEq} / \mathrm{L}$ & 29,5 & 3 \\
\hline $\begin{array}{l}\text { SS } 0.9 \% 400 \text { cc } \\
(62 \mathrm{mEq})+100 \mathrm{cc} \\
\text { (200 mEq) Cloruro } \\
\text { de sodio al } 10 \%\end{array}$ & $524 \mathrm{mEq} / \mathrm{L}$ & 30,7 & 3,1 \\
\hline $\begin{array}{l}\text { SS 0.9\% } 455 \text { cc } \\
(70 \mathrm{mEq})+45 \mathrm{cc} \\
(180 \mathrm{mEq}) \text { Cloruro } \\
\text { de sodio al } 20 \%\end{array}$ & $500 \mathrm{mEq} / \mathrm{L}$ & 29,3 & 2,9 \\
\hline $\begin{array}{l}\text { SS 0.9\% } 410 \text { cc } \\
(63 \mathrm{mEq})+90 \mathrm{cc} \\
(180 \mathrm{mEq}) \text { Cloruro } \\
\text { de sodio al } 10 \%\end{array}$ & $486 \mathrm{mEq} / \mathrm{L}$ & 28,4 & 2,8 \\
\hline $\begin{array}{l}\text { SS 0.9\% } 500 \text { cc } \\
(77 \mathrm{mEq})+45 \mathrm{cc} \\
(180 \mathrm{mEq}) \text { Cloruro } \\
\text { de sodio al } 20 \%\end{array}$ & $472 \mathrm{mEq} / \mathrm{L}$ & 27,6 & 2,8 \\
\hline
\end{tabular}

Fuente: autores. Agradecimiento al Dr. Jorge Mario Palmezano por concepto original.

\section{b. Hiponatremia hipovolémica}

En este caso, se debe realizar una corrección de la depleción con uso de solución salina al o,9\% o Lactato de Ringer. Hay que tomar en cuenta que en estos pacientes, la corrección del volumen produce una interrupción abrupta de la liberación de vasopresina por lo cual existe un riesgo elevado de sobrecorrección ${ }^{33}$. Existen algunas variables que preceden a este fenómeno como son un aumento del volumen urinario y una disminución de la osmolaridad urinaria ${ }^{1,33}$. Otra medida útil para evitar 
este fenómeno es realizar una corrección más lenta del volumen en los casos que así se pudiera, por ejemplo, en ausencia de choque o grados elevados de deshidratación.

Tabla 4. Manejo de reposición de sodio en hiponatremia.

\begin{tabular}{|c|c|}
\hline $\begin{array}{l}\text { Temporalidad/ } \\
\text { Síntomas }\end{array}$ & Reposición recomendada con SS3\% \\
\hline $\begin{array}{l}\text { Aguda } \\
\text { sintomática }\end{array}$ & $\begin{array}{l}\text { SS3\% } 100 \text { - } 150 \mathrm{~mL} \text { en } 10 \text { a } 20 \text { minutos. } \\
\text { Repetir hasta } 3 \text { veces, hasta mejoría de } \\
\text { síntomas o ascenso 4-6mEq/L en } 1 \text { hora } \\
\text { Alternativa: infusión de } \mathrm{SS} 3 \% 2-4 \mathrm{ml} / \mathrm{Kg} / \mathrm{h} \text { por } \\
\text { 2-3 horas o hasta mejoría de los síntomas }\end{array}$ \\
\hline $\begin{array}{l}\text { Crónica } \\
\text { sintomática }\end{array}$ & $\begin{array}{l}\text { Síntomas severos: igual que en hiponatremia } \\
\text { aguda } \\
\text { Síntomas moderados: } 1-2 \mathrm{~mL} / \mathrm{Kg} / \mathrm{h} \text {, para una } \\
\text { tasa promedio de aumento } 1-2 \mathrm{mmol} / \mathrm{L} / \mathrm{h} \\
\text { Síntomas leves: } 0,5 \mathrm{~mL} / \mathrm{Kg} / \mathrm{h} \text {, para una tasa } \\
\text { promedio de aumento } 0,5 \mathrm{mEq} / \mathrm{L} / \mathrm{h}\end{array}$ \\
\hline
\end{tabular}

Fuente: autores.

\section{c. Hiponatremia euvolémica}

La mayoría de estos pacientes corresponden a cuadros de Síndrome de secreción inadecuada de $\mathrm{ADH}$, por lo que la búsqueda y eliminación de la causa es clave en el éxito del tratamiento ${ }^{11,32}$. De manera concomitante, se puede usar la restricción de agua libre al día. Una forma sencilla de estimar el valor meta es el ratio entre la suma del sodio y potasio urinario sobre la concentración de sodio sérico. Los valores superiores a 1 requiere restricción hídrica estricta (menor a $500 \mathrm{cc}$ al día) mientras que valores menores a 1 , pueden tener una restricción más tenue (menor a $1000 \mathrm{cc}$ al día) $)^{1,28}$.

Es necesario resaltar sin embargo que la mayoría de los pacientes persisten con hiponatremia a menos que se corrija la causa, razón por la cual otras medidas coadyuvantes pueden ser usadas. En primer lugar, los vaptanes en sus dos formas disponibles: conivaptan (antagonista no selectivo de receptor de vasopresina V1-V2) y el tolvaptán (antagonista selectivo V2). En promedio, estos medicamentos ascienden entre un 5-8 mEq/L de sodio a lo largo de algunos días. Sin embargo, no han demostrado utilidad como medicamento crónico ni en casos agudos severos y tienen un elevado costo ${ }^{32}$.
Por el contrario, la urea ha demostrado utilidad para elevación de alrededor de $6 \mathrm{mEq} / \mathrm{L}$ luego de 4-5 días de uso pero posee poca evidencia clínica y no hay estudios de comparación con vaptanes'. En suma, tanto los vaptanes como la úrea pueden ser usados en casos de hiponatremia no severos refractarios al manejo médico pero la evidencia actual es limitada.

\section{Conclusiones}

La hiponatremia es el trastorno electrolítico más común en la práctica clínica, razón por la cual el conocimiento para el abordaje inicial en urgencias es clave para un enfoque diagnóstico y terapéutico adecuado. Las manifestaciones clínicas son muy diversas, pero debe tomarse en cuenta en pacientes con alteración de la conciencia o con factores de riesgo para su presentación. Una buena historia clínica debe generar una idea del tiempo de evolución y la etiología, descartando causas secundarias como medicamentos o exceso en la ingesta de líquidos. Adicionalmente, la realización de estudios en sangre y orina permiten una adecuada clasificación para determinar el mejor protocolo en búsqueda de etiología y los mecanismos subyacentes. En cuanto al manejo, este dependerá en gran medida de la velocidad de instauración y la causa, en general se realiza una corrección con solución salina hipertónica de manera lenta para no generar SDO con seguimiento estricto de los niveles séricos de sodio para ajustar la tasa de infusión y evitar sobrepasar las metas recomendadas.

\section{Referencias bibliográficas}

1. Seay NW, Lehrich RW, Greenberg A. Diagnosis and Management of Disorders of Body Tonicity-Hyponatremia and Hypernatremia: Core Curriculum 2020. Am J Kidney Dis. 2020;75(2):272-86.

2. Waikar SS, Mount DB, Curhan GC. Mortality after Hospitalization with Mild, Moderate, and Severe Hyponatremia. Am J Med. 2009;122(9):857-65.

3. Liamis G, Rodenburg EM, Hofman A, Zietse R, Stricker BH, Hoorn EJ. Electrolyte Disorders in Community Subjects: Prevalence and Risk Factors. Am J Med. 2013;126(3):256-63.

4. Mannesse CK, Vondeling AM, van Marum RJ, van Solinge WW, Egberts TCG, Jansen PAF. Prevalence of hyponatremia on geriatric wards compared to other settings over four decades: A systematic review. Ageing Res Rev. 2013;12(1):165-73.

5. Burguera V, Rodríguez-Palomares JR, Fernández-Codejón O, Tenorio MT, Rey JM del, Liaño F. Epidemiología de la hiponatremia. Nefrología. 2011;2(2):1-83.

6. Ganguli A, Mascarenhas RC, Jamshed N, Tefera E, Veis JH. Hyponatremia: incidence, risk factors, and consequences in the elderly in a home-based primary care program. Clin Nephrol. 2015;84(8):75-85.

7. EJ,Zietse R. Diagnosis and Treatment of Hyponatremia:Compilation of the Guidelines. J Am Soc Nephrol. 2017;28(5):1340-9.

8. Godoy DA, Álvare E, Campi V, Soler C, Masotti L, Di Napoli M. Enfoque práctico para el diagnóstico y tratamiento de los estados 
poliúricos en pacientes con injuria cerebral aguda. Rev Med Chil. 2013;141(5):616-25.

9. Adrogué HJ, Madias NE. The Challenge of Hyponatremia. J Am Soc Nephrol. 2012;23(7):1140-8.

10. Zieg J. Pathophysiology of Hyponatremia in Children. Front Pediatr. 2017;5(x)

11. Braun MM, Barstow CH, Pyzocha NJ. Diagnosis and management of sodium disorders: hyponatremia and hypernatremia. Am Fam Physician. 2015;91(5):299-307.

12. Jeppesen AN, Jensen HK, Donskov F, Marcussen N, von der Maase H. Hyponatremia as a prognostic and predictive factor in metastatic renal cell carcinoma. Br J Cancer. 2010;102(5):867-72.

13. Tzoulis P, Bagkeris E, Bouloux P-M. A case-control study of hyponatraemia as an independent risk factor for inpatient mortality. Clin. Endocrinol (Oxf). 2014;81(3):401-7.

14. Holland-Bill L, Christiansen CF, Heide-Jørgensen U, Ulrichsen SP, Ring T, Jørgensen JOL, et al. Hyponatremia and mortality risk: a Danish cohort study of 279508 acutely hospitalized patients. Eur. J. Endocrinol. 2015;173(1):71-81.

15. Hoorn EJ, Spasovski G. Recent developments in the management of acute and chronic hyponatremia. Curr Opin Nephrol Hypertens. 2019;28(5):424-32.

16. Verbalis JG. Disorders of water balance. EN: Duffy NA, coordinador.. Brenner and Rector's The Kidney. 11a ed. Canadá: Elsevier; 2019. p. 443-95.

17. Gunathilake R, Oldmeadow C, McEvoy M, Kelly B, Inder K, Schofield P, et al. Mild hyponatremia is associated with impaired cognition and falls in community-dwelling older persons. J Am Geriatr Soc. 2013;61(10):1838-9.

18. Chung MC, Yu TM, Shu KH, Wu MJ, Chang CH, Muo CH, et al. Hyponatremia and increased risk of dementia: A population-based retrospective cohort study. PLoS One. 2017;12(6):1-11.

19. Shavit L, Mikeladze I, Torem C, Slotki I. Mild hyponatremia is associated with functional and cognitive decline in chronic hemodialysis patients. Clin Nephrol. 2014;82(5):313-9.

20. Henry DA. In the clinic: Hyponatremia. Ann Intern Med. 2015;163(3):ITC1-ITC19.
21. Buffington MA, Abreo K. Hyponatremia: A Review. J Intensive Care Med. 2016;31(4):223-36.

22. Adrogué HJ, Madias NE. Hyponatremia. N Engl J Med. 2000;342(21):1581-9.

23. Spasovski G, Vanholder R, Allolio B, Annane D, Ball S, Bichet D, et al. Clinical practice guideline on diagnosis and treatment of hyponatraemia. Eur. J. Endocrinol. 2014;170(3):1-47.

24. Pedrós C, Arnau JM. Hiponatremia y SIADH por medicamentos. Rev Esp Geriatr Gerontol. 2010;45(4):229-31.

25. Hoorn EJ, Zietse R. Diagnosis and treatment of hyponatremia: Compilation of the guidelines. J Am Soc Nephrol. 2017;28(5):1340-9.

26. Musch W, Thimpont J, Vandervelde D, Verhaeverbeke I, Berghmans T, Decaux G. Combined fractional excretion of sodium and urea better predicts response to saline in hyponatremia than do usual clinical and biochemical parameters. Am J Med. 1995;99(4):34855.

27. Chung H-M, Kluge R, Schrier RW, Anderson RJ. Clinical assessment of extracellular fluid volume in hyponatremia. Am J Med. 1987;83(5):905-8. Sterns RH. Disorders of Plasma Sodium - Causes, Consequences, and Correction. N Engl J Med. 2015;372(1):55-65.

28. Sterns RH. Disorders of Plasma Sodium - Causes, Consequences, and Correction. N Engl J Med. 2015;372(1):55-65

29. Verbalis JG, Goldsmith SR, Greenberg A, Korzelius C, Schrier RW, Sterns RH, et al. Diagnosis, evaluation, and treatment of hyponatremia: Expert panel recommendations. Am J Med. 2013;126(10 Suppl 1):1-42.

30. Sterns RH. Treatment of Severe Hyponatremia. Clin J Am Soc Nephrol. 2018;13(4):641-9.

31. Lambeck J, Hieber M, Dreßing A, Niesen W-D. Central pontine myelinolysis and osmotic demyelination syndrome. Dtsch Aerztbl Int. 2019;116:600-6.

32. Ball SG, Iqbal Z. Diagnosis and treatment of hyponatraemia. Best Pract Res Clin Endocrinol Metab. 2016;30(2):161-73.

33. Rondon-Berrios H, Agaba EI, Tzamaloukas AH. Hyponatremia: pathophysiology, classification, manifestations and management. Int Urol Nephrol. 2014;46(11):2153-65. 